\title{
ACOUSTIC SOURCE TERM FORMULATIONS IN THE CAVITY TONE
}

\author{
PÉTER TAMÁs NAGY \\ Department of Hydrodynamic Systems, Budapest University of Technology and Economics \\ Budapest, H-1111, Hungary \\ pnagy@hds . bme.hu \\ ANDREAS HÜPPE \\ Institute of Mechanics and Mechatronics, Vienna University of Technology \\ Vienna, A-1060, Austria \\ andreas.hueppe@tuwien.ac .at \\ Manfred Kaltenbacher \\ Institute of Mechanics and Mechatronics, Vienna University of Technology \\ Vienna, A-1060, Austria \\ manfred.kaltenbacher@tuwien.ac.at \\ GYÖRGY PAÁL \\ Department of Hydrodynamic Systems, Budapest University of Technology and Economics \\ Budapest, H-1111, Hungary \\ paal@hds.bme.hu
}

[Received: March 8, 2016, Accepted: August 22, 2016]

\begin{abstract}
The cavity tone at low speed is simulated by a hybrid aeroacoustic approach. Thereby, a rectangular, open cavity with a length-to-depth ratio 2 is investigated at a Mach number of about 0.05 . We compared various source formulations (velocity, pressure, divergence of Lighthill tensor, Lamb vector) to each other. The simulations were carried out by ANSYS CFX and the in-house code CFS++. The results indicate that velocity based formulations provide better results.
\end{abstract}

Mathematical Subject Classification: 35E99

Keywords: cavity tone, CAA, CFD, source term, low Mach number, implicit damping in CFX

\section{INTRODUCTION}

In this paper cavity tone is investigated, which is one of the fundamental aeroacoustic flow configurations (Figure 1). The "cavity" is a rectangular cutout in a surface, typically a plane. If there is flow over the cavity, a shear layer is formed, which can lose its stability. The instability wave propagates from the leading edge to the downstream (trailing) edge, while the amplitude of the wave continuously increases; thus, 
a feedback mechanism develops between the leading and trailing edge. The pressure fluctuation at the trailing edge perturbs the shear layer at the leading edge, where the previously mentioned instability waves are generated. This feedback mechanism leads to oscillations that are sometimes even audible. Apart from the generated sound, this phenomenon usually leads to unwanted mechanical vibrations and also to increased drag force.

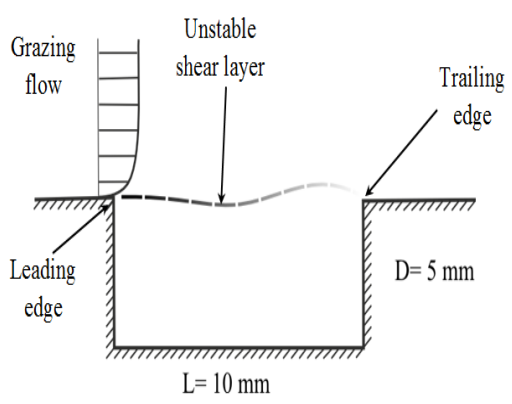

Figure 1. The investigated configuration of cavity-tone

The first studies on cavity noise concerned aeronautic applications in the 1950-1960s [1. The motivation of research was that the oscillations appeared in the weapon bays of military aircraft. This oscillation can excite the vibrational modes of the aircraft structure, which can quickly lead to structural fatigue issues inside the aircraft [2] and can significantly increase the drag force, too. The speed of the flow was high subsonic to supersonic in these cases. The need to investigate configurations at lower velocities appeared in the 1970s mainly because aircraft wheel wells were seen to be an important source of aerodynamic noise during the landing and take-off of airplanes [3, 4. In the meantime many other technical applications have been discovered: door gaps and sunroofs in automobiles, closed side branches in gas pipelines, slotted flumes, slotted wall wind and water tunnels, bellows-type pipe geometries [5, canal locks, harbor entries and gaps between wagons or the pantograph of trains [6].

In this paper a fundamental open cavity with length-to-depth ratio of 2 is investigated at a flow speed of about $0.05 \mathrm{Ma}$ (Mach-number, defined as the ratio of far field velocity of the flow and the speed of sound). At such a low Mach number the disparity of scales of the acoustic and the flow field is significant. The grid of the flow simulation must be fine enough to resolve the boundary layer and small vortices to provide sufficiently accurate results. Usually the desired grid resolution for acoustic computation can be much coarser because the acoustic wavelength is much larger than the vortices or even the cavity. In addition, there are several orders of magnitude differences in the aerodynamic and acoustic variables. E.g. a $1 \mathrm{~m} / \mathrm{s}$ velocity fluctuation is not extreme in flows, while the amplitude of the acoustic velocity fluctuation at a particle velocity level of $80 \mathrm{~dB}$ is only $0.5 \mathrm{~mm} / \mathrm{s}$. The human hearing threshold at $1 \mathrm{kHz}$ is around $0 \mathrm{~dB}$ sound pressure level, corresponding to $20 \mu \mathrm{Pa}$ 
in air, which is again several orders of magnitude smaller than an average pressure fluctuation in aerodynamics $(10-100 \mathrm{~Pa})$. These orders of magnitude differences between the variables increase the numerical noise, which can spoil the whole acoustic simulation [7]. To reduce these errors it is typical to separate the acoustic and the aerodynamic flow fields. The common name for these approaches is hybrid Computational AeroAcoustics (CAA). If the two fields are separated, the crucial point of the computation is to determine the coupling between them. The idea is to derive equations for the acoustic field (model the acoustic field) and then to define source terms, which are based on the independently solved flow field. The optimal formulation of these equations and source terms is still under research. In this paper various source terms are investigated for the acoustic wave equation. Some of them are valid only for incompressible fluid. The compressibility in our cavity configuration does not play an important role, allowing us to carry out incompressible flow simulations and to use coupling formulations that are valid only for incompressible flow. At the same time a compressible flow simulation was also carried out to verify the previous statement and to investigate the effect of compressibility to the sources.

In Section 2 the governing equations of fluid dynamics are presented briefly and based on them the various formulations of the source terms are introduced. In Section 3 the CFD simulations are described and their acoustic source fields are compared to each other. In Section 4 the acoustic simulations are presented. Finally, in Section 5 we make some concluding remarks.

\section{SOURCE TERM FORMULATION}

2.1. The acoustic wave equation with general sources. The basic equations, which describe both the flow and acoustic field, are the conservation equations of mass, momentum and energy

$$
\begin{gathered}
\frac{\partial \rho}{\partial t}+\nabla \cdot(\rho \underline{\mathbf{u}})=0, \\
\frac{\partial(\rho \underline{\mathrm{u}})}{\partial t}+\nabla \cdot(\rho \underline{\mathrm{u}} \otimes \underline{\mathrm{u}})=\nabla \cdot \underline{\underline{\mathrm{T}}}, \\
\frac{\partial \rho e}{\partial t}+\nabla \cdot(\rho \underline{\mathrm{u}})=\nabla \cdot(-p \underline{\mathrm{u}}+\underline{\tau \underline{\mathrm{u}}})+\nabla \cdot(\lambda \nabla T),
\end{gathered}
$$

where $\rho$ is the density, $t$ is the time, $\nabla$ is the "nabla" operator, $\underline{u}$ is the velocity vector, $\underline{\underline{T}}$ is the stress tensor, $e$ is the total energy, $p$ is the pressure, $\underline{\underline{\tau}}$ is the viscous stress $\overline{\overline{\text { tensor, }}} T$ is the temperature. The following expressions hold if $\overline{\bar{t}}$ the fluid is Newtonian (equation (2.4) ) and an ideal gas is assumed (equation (2.5))

$$
\underline{\underline{\mathrm{T}}}=-\left(p+\frac{2}{3} \mu \nabla \cdot \underline{u}\right) \underline{\underline{\mathrm{I}}}+\mu\left(\nabla \otimes \underline{\mathrm{u}}+(\nabla \otimes \underline{\mathrm{u}})^{T}\right),
$$




$$
e=\frac{p}{\rho(\gamma-1)}+\frac{u^{2}}{2}
$$

where $\mu$ is the dynamic viscosity, $\underline{I}$ is the identity tensor. Now let us split the variables into mean (time averaged) and fluctuating components as

$$
f(t, x)=\bar{f}(x)+f^{\prime}(t, x),
$$

and neglect the viscous and non-linear terms. Then the following expression can be obtained

$$
\begin{gathered}
\frac{1}{c_{0}^{2}} \frac{\partial p^{\prime}}{\partial t}+\nabla \cdot \rho \underline{\mathrm{u}}^{\prime}=\Phi_{\text {cont }}, \\
\frac{\partial \rho \underline{\mathrm{u}}^{\prime}}{\partial t}+\nabla p^{\prime}=\underline{\Phi}_{m o m} .
\end{gathered}
$$

where $\Phi_{c o n t}$ and $\underline{\Phi}_{m o m}$ are general sources for the equations and $c_{0}$ is the speed of sound.

Let us take the time derivative of (2.7) and the divergence of (2.8), and assume the fluctuating variables to be purely acoustic, denoted by superscript "a" (see 8 ] for details). Then the velocity can be eliminated and we get the well-known wave equation for the acoustic pressure with the sources $(S)$ on the right-hand side

$$
\frac{1}{c_{0}^{2}} \frac{\partial^{2} p^{a}}{\partial t^{2}}-\Delta p^{a}=\underbrace{\frac{\partial}{\partial t} \Phi_{c o n t}-\nabla \cdot \underline{\Phi}_{m o m}}_{\mathrm{S}} .
$$

2.2. The Lighthill analogy. The first, most famous and still widely used analogy is the one proposed by Lighthill in 1951 9]. The basic idea of Lighthill was that the terms, neglected during the derivation (linearization) of the wave equation, act as sources for the acoustic equation, i. e.

$$
\mathrm{S}=\nabla \cdot \underline{\Phi}_{m o m}=\nabla \cdot \nabla \cdot \underline{\underline{\mathrm{T}}}_{L H} \cdot
$$

The original form of the Lighthill-tensor is

$$
\underline{\underline{T}}_{L H}=\rho \underline{\mathrm{u}} \otimes \underline{\mathrm{u}}+p^{\prime} \underline{\underline{\mathrm{I}}}-\underline{\underline{\tau}}-\mathrm{c}_{0}^{2}\left(\rho-\rho_{0}\right) \underline{\underline{\mathrm{I}}} .
$$

where $\rho_{0}$ is the ambient density. Lighthill assumed that if the viscous terms are small (the Reynolds-number is high), the process is close to isentropic and the main sources of sound are vortices, then the tensor can be approximated by

$$
\underline{\mathrm{T}}_{L H} \approx \rho \underline{\mathrm{u}} \otimes \underline{\mathrm{u}} .
$$

In what follows, we shall also employ the above simplification.

2.3. Approximation of the Lighthill analogy. The following approximations of the Lighthill tensor can be found in [8]. 
2.3.1. The Lamb vector. Let us decompose the velocity field according to Helmholtz into an irrotational and a solenoidal vector field. If we assume that the Lighthill source term is based on the incompressible velocity the following relation holds

$$
\mathrm{S}=\nabla \cdot \nabla \cdot\left(\rho \underline{\mathrm{u}}^{i c} \otimes \underline{\mathrm{u}}^{i c}\right)=\nabla \cdot\left(\rho \underline{\mathrm{\omega}} \times \underline{\mathrm{u}}^{i c}\right)+\nabla \cdot \nabla\left(\frac{1}{2} \rho \underline{\mathrm{u}}^{i c} \cdot \underline{\mathrm{u}}^{i c}\right),
$$

where $u^{i c}$ is the solenoidal part of the velocity after Helmholtz decomposition.

2.3.2. The Laplacian of the pressure. The Lighthill tensor can be approximated by the incompressible pressure in the following way. Let us neglect the viscous terms in equation 2.2 and take its divergence, which leads to

$$
\nabla \cdot\left(\frac{\partial(\rho \underline{\mathrm{u}})}{\partial t}\right)+\nabla \cdot \nabla \cdot(\rho \underline{\mathrm{u}} \otimes \underline{\mathrm{u}})=-\Delta p .
$$

In an incompressible fluid the first term on the left-hand side is zero because of the incompressible continuity equation and the symmetry property of the spatial and temporal derivatives. The second term on the left-hand side is the approximation of the previously mentioned Lighthill tensor,

$$
\nabla \cdot \nabla \cdot \underline{\underline{T}}_{L H} \approx \nabla \cdot \nabla \cdot(\rho \underline{\mathrm{u}} \otimes \underline{\mathrm{u}}) \approx-\Delta p .
$$

This relation is valid only, if the viscous terms are negligible, i.e. the Reynolds-number is high.

2.3.3. The time derivative of the pressure. The following source term formulation and a detailed, exact derivation of it can be found in 7], we give here only a short, rudimentary derivation. If a quiescent medium is assumed,equation (2.7) is still valid for fluctuating variables. First, let us split the fluctuating pressure into acoustic and incompressible pressure. Then, we take the time derivative and rearrange the incompressible pressure to the right-hand side. This term can be conceived as the source term for the continuity equation,

$$
\Phi_{\text {cont }}=-\frac{1}{c_{0}^{2}} \frac{\partial p^{i c}}{\partial t}
$$

If we substitute into equation (2.9), the source term can be calculated as

$$
S=\frac{\partial \Phi_{\text {cont }}}{\partial t}=-\frac{1}{c_{0}^{2}} \frac{\partial^{2} p^{i c}}{\partial t^{2}} .
$$

\section{CFD Simulations AND ACOUSTIC LOAD}

3.1. The CFD simulation domain. In the first step 2D CFD simulations were performed using ANSYS CFX. The cavity dimensions were the following: the length of the cavity was $\mathrm{L}=10 \mathrm{~mm}$; the depth of the cavity was $D=5 \mathrm{~mm}$. The simulation domain was defined to be a length of $2 L$ before the cavity, a length of $3 L$ after the cavity and $3 \mathrm{~L}$ above the cavity. A sponge layer around the simulation domain was 
necessary in the compressible simulation to avoid reflection at the boundaries. The thickness of this layer was $4.5 \mathrm{~L}$ in both horizontal and vertical directions. The mesh was generated by ANSYS ICEM. The finest mesh resolved the cavity by 166x106 cells. The monitor point was placed $0.07 \mathrm{D}$ below the corner on the rear cavity wall, which is the most favorable place (experimentally measurable, smaller residuals, larger pressure signal) for the investigation (see [1, 10]).

Table 1. The main parameters of the CFD simulations

\begin{tabular}{|c|c|}
\hline Name & Value \\
\hline Mach number & 0.05 \\
\hline Far-field velocity & $17.306 \mathrm{~m} / \mathrm{s}$ \\
\hline Length of cavity & $10[\mathrm{~mm}]$ \\
\hline Depth of cavity & $5[\mathrm{~mm}]$ \\
\hline Boundary layer thickness at leading edge & $1.22[\mathrm{~mm}]$ \\
\hline Kinematic viscosity & $1.545 \times 10^{-5}\left[\mathrm{~m}^{2} / \mathrm{s}\right]$ \\
\hline Reynolds number $\left(\mathrm{Re}_{L}\right)$ & $\sim 11200$ \\
\hline Density & $1.185\left[\mathrm{~kg} / \mathrm{m}^{3}\right]$ \\
\hline Reference temperature & $25\left[{ }^{\circ} \mathrm{C}\right]$ \\
\hline Flow model & Laminar flow was assumed \\
\hline
\end{tabular}

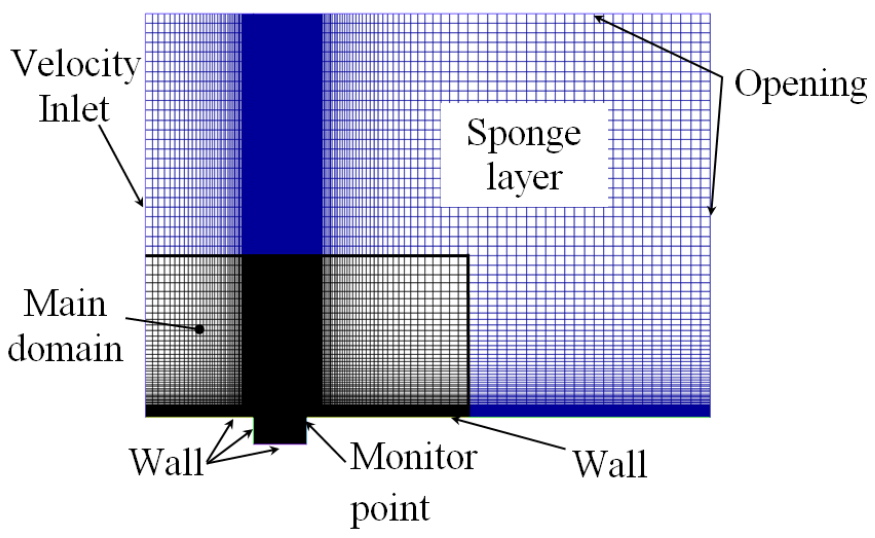

Figure 2. The CFD domain

The CFD domain with the applied boundary conditions can be seen in Figure 2 . The velocity inlet boundary condition (BC) was a prescribed velocity profile. This velocity profile was calculated by an in-house MatLab code that generates the Blasius profile. This code provides the prescribed value of boundary layer thickness at the 
leading edge. It was $1.22 \mathrm{~mm}$ in these simulations. The walls at the bottom were modeled as adiabatic "no-slip walls". At the outlet and the top of the domain the so-called "opening" boundary condition was used [11, which prescribes the relative pressure $(0 \mathrm{~Pa})$ and the flow direction (normal to the boundary). However, if the pressure is rigorously constant in a compressible simulation, the acoustic waves are reflected from the boundary. The solution to avoid such a problem will be discussed in the next subsection. The advection scheme was set to "High Resolution" (second order scheme) while second order backward Euler transient scheme was applied. Two constant timestep sizes were applied: $1 e^{-6} \mathrm{~s}$ and $2.5 e^{-7} \mathrm{~s}$, meaning the rms CourantFriedrich-Levy number were around 0.53 and 0.14 , respectively. Between the two results no significant difference was obtained. During the solution the convergence criteria for the iteration was that the root mean square of the residuals had to be smaller than $10^{-5}$.

Table 2. Mesh parameters of the CFD simulations

\begin{tabular}{|c|c|c|c|}
\hline Case & Resolution of cavity & Smallest element edge length & Number of elements \\
\hline Coarse & $141 \times 71$ & $20 \mu \mathrm{m}$ & $\sim 104000$ \\
\hline Medium & $151 \times 91$ & $7 \mu \mathrm{m}$ & $\sim 133000$ \\
\hline Fine & $166 \times 106$ & $3.5 \mu \mathrm{m}$ & $\sim 174000$ \\
\hline
\end{tabular}

The mesh investigations in the classical CFD sense were already carried out by Farkas et al. in [12] and [10] on the same configuration. The meshes were investigated in this work too, because the resolution of the CFD grid is usually not sufficient for acoustic coupling. Mesh refinement was applied only close to the trailing edge (the main acoustic source), which differs from the usual uniform method, because in the aerodynamic sense the mesh was already appropriate. The comparison was made based on the original Lighthill source term formulation only and the main parameters of the investigated meshes can be found in Table 1. The observations are the following: the difference in the results between the coarse and medium mesh is significant. The main acoustic source is not well resolved on the coarse grid. Further improvement is hardly noticeable between the medium and the fine mesh. Based on these results, the medium mesh is selected for further simulations.

3.2. Reflection avoidance in compressible CFD simulations. In CFX there is a beta option to turn on acoustic non-reflectivity at the boundaries but it works well only if the sound waves hit the boundary in the normal direction. (Non-reflective boundary conditions are implemented in most commercial codes based on the $1 \mathrm{D}$ wave equation [13.) If the perturbation velocity has also a tangential component at the boundaries, the non-reflective boundary condition can cause instabilities or reflect the acoustic waves [10. Other passive possibilities to avoid reflections are to artificially increase the viscosity close to the boundaries or to stretch the grid there. These options are infeasible because of presence of the wall behind the cavity. Further possibilities are the active damping methods: implicit damping, explicit damping, artificial 
convection and perfectly matched layer. With the exception of implicit damping these methods cannot be implemented in a commercial code like CFX. The explicit technique needs a writing access to the state vectors, which is prohibited in CFX, while the artificial convection and the perfectly matched layer method require changing the governing equations significantly, which is again not feasible in commercial software. Furthermore the perfectly matched layers technique is not developed for the NavierStokes equations, according to authors' knowledge. In our case the implicit damping method was implemented in CFX for the continuity equation. It can be achieved by the addition of the following source terms to the governing equations

$$
S_{C}=-\sigma_{1}\left(p-p_{\text {mean }}\right),
$$

where $S_{C}$ is the source terms of continuity equation, $\sigma_{1}$ is the amplitude function of the damping, $p$ is the relative pressure, $p_{\text {mean }}$ is the prescribed value at any given grid point, in our case it was $0 \mathrm{~Pa}$.

In the next step $\sigma_{1}$ has to be specified and based on [14] we apply

$$
\sigma_{1}=\hat{\sigma}\left(\frac{x-x_{0}}{H_{s}-x_{0}}\right)^{\beta}
$$

where $\hat{\sigma}$ depends only on non-geometrical variables and the other term provides the geometrical shape function, $\mathrm{x}$ is the running coordinate, $x_{0}$ is the location of the beginning of the sponge layer, $H_{s}$ is the location of the end of the sponge layer, $\beta$ is the shape parameter. $\beta$ was chosen between 2 and 2.5 in [13, while in other papers it was 3 [15]. Here it was chosen to be 2, a similar formula can be used in the y (or in $3 \mathrm{D}$ simulation in the $\mathrm{z}$ ) direction.

A new expression was derived for $\hat{\sigma}$ because no suggestion was found in literature to choose it. Let us write the equation of state of the ideal gas

$$
\rho=\frac{p_{a b s}}{\gamma R T}
$$

where $\rho$ is the density, $p_{a b s}$ is the absolute pressure, $\gamma$ is the specific heat ratio and $R$ is the gas constant. Now we split the variables into mean and perturbation part while assuming constant temperature. It is trivial that the fluctuations of the relative and absolute pressure are the same because of constant difference.

$$
\left(\bar{\rho}+\rho^{\prime}\right)=\frac{\left(\overline{p_{a b s}}+p^{\prime}\right)}{\gamma R T} .
$$

The mean variables must satisfy the equation of state, thus they can be subtracted, which leads to

$$
\rho^{\prime}=\frac{p^{\prime}}{\gamma R T} .
$$


The mass flow rate per unit volume can be approximated as

$$
\hat{\sigma} \propto \frac{\rho^{\prime}}{\Delta t}
$$

where $\Delta t$ is the time needed for pressure fluctuation to propagate through one cell, i.e.

$$
\Delta t=\frac{\Delta x}{v_{\text {prop }}}
$$

Here, $\Delta x$ is the cell size in the direction of the propagation, $v_{\text {prop }}$ is the propagation velocity of the pressure disturbance, which can be of various physical origin in a complex flow. The propagation velocity of the acoustic waves is the speed of sound but in the flow other pressure fluctuations exist: for example the instability waves along the shear layer or the propagating vortices after the cavity. The speed of these fluctuations is obviously much less than the acoustic speed so that $v_{\text {prop }}$ must be chosen carefully. Finally, equations (3.5)- $(3.7)$ lead to following expression:

$$
\hat{\sigma} \propto \frac{p^{\prime}}{\gamma R T} \frac{v_{\text {prop }}}{\Delta x}=\frac{p^{\prime}}{c_{0}^{2}} \frac{v_{\text {prop }}}{\Delta x}=\frac{p^{\prime}}{c_{0}} \frac{M a_{\text {prop }}}{\Delta x},
$$

where $M a_{\text {prop }}$ is the Mach number based on the propagation velocity. If $\hat{\sigma}$ is greater than the right-hand side of (3.8), the sponge layer is overdamped, causing stability problems. During the time the pressure fluctuation passes the cell, its value can have opposite sign and the magnitude can be larger than its value when it entered the cell. In the simulations, the following expression was used, based on (3.8)

$$
\hat{\sigma}=C_{1}\left(M a_{\text {prop }}\right) \frac{p^{\prime}}{c_{0}} \frac{1}{\Delta x},
$$

where $C_{1}$ was the constant with which the sponge layer can be tuned. Its allowable maximal value is 1 in the case of pure acoustic waves (above the cavity), and it has to be decreased if other types of pressure fluctuations are present in the sponge layer (after the cavity).

3.3. The results of CFD simulations. Fast Fourier Transformation (FFT) was carried out on the pressure signals at the monitor point in the incompressible and the compressible simulations and the results can be seen in Figure 3 . The peaks are practically the same. The fundamental frequency is $1522 \mathrm{~Hz}$ with the amplitude of $26.4 \mathrm{~Pa}(122.4 \mathrm{~dB})$ in the incompressible simulation, while in the compressible simulation the fundamental frequency is $1530 \mathrm{~Hz}$ and the amplitude was $26.9 \mathrm{~Pa}(122.6$ $\mathrm{dB})$. Although the pressure signal in the compressible simulation was a bit noisier, there was technically no difference between the two simulations. The compressibility of the fluid thus can be neglected, as expected. 


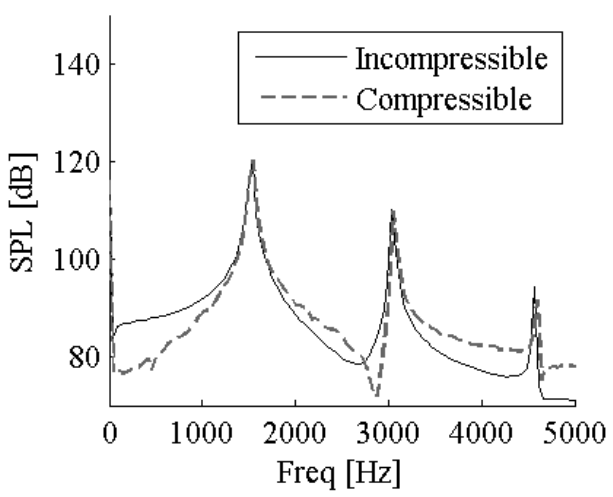

Figure 3. The FFT of the pressure signal at the monitor point

3.4. The comparison of various source term formulations. In the next step the sources were calculated for the acoustic simulation, based on the CFD results. The sources introduced in Section 2 are compared with each other. In the first investigation the source field based on the Lighthill tensor was compared to the Laplacian of the pressure in the incompressible case because the previously introduced pressure based formulations are valid only for incompressible flow simulations. The time signal compared inside the cavity can be seen in Figure 4. The monitoring point was placed in the shear layer at equal distances far from the edges of the cavity. To be precise, the monitoring point is at $x=L / 2, y=0$, where the origin is the leading edge and the $\mathrm{x}$ direction is the main flow direction.

In Figure 4 it can be clearly seen that the Laplacian of the pressure approximates well the second derivative of the Lighthill tensor but the signal was noisier in the case of the pressure-based-formulation. The reason for this phenomenon can be that the pressure values themselves suffer from higher numerical error, which is further magnified during the calculation of second spatial derivatives. It is difficult to interpret the results because of the lack of knowledge about how CFX calculates the pressure.

In the next step an FFT is performed on the various sources (Figure 5). Here, the spatial distributions (the shapes) of the sources were compared. The Laplacian of the pressure was excluded from the comparison because no significant difference could be observed between Laplacian of the pressure and the double divergence of the Lighthill tensor. Only a small difference was recognized in the compressible computations very close to the edge. The results of the comparison are summarized in Table 2 for the incompressible simulations. First, the effect of the mean value $\left(F_{\text {Mean }}\right)$ of the acoustic load is investigated at a probe location close to the trailing edge in all cases. The zero frequency component was compared to the fundamental mode component $\left(F_{1 s t}\right)$. It is probable that this value highly depends on the probe location but the tendencies can be roughly estimated based on the monitoring point. This ratio was high in the case of velocity-based source terms (Lighthill tensor, Lamb vector) and was low in 


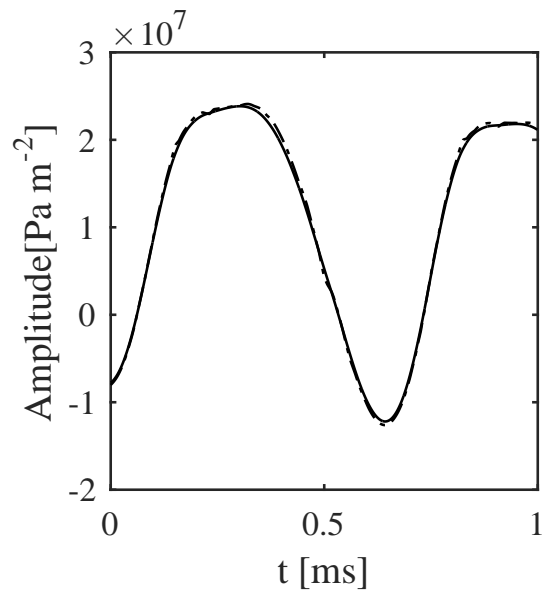

(a)

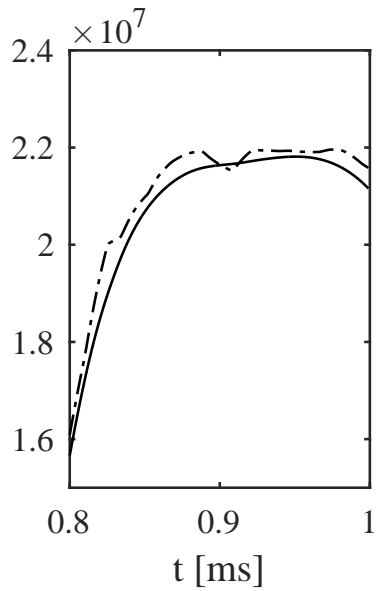

(b)

Figure 4. Comparison of the sources calculated based on the Lighthill-tensor (continuous line) and the Laplacian of the pressure (dashed line) in the middle of the shear layer

the case, when the source was calculated based on the time derivative of the pressure. The high mean value is non-acoustical (only the fluctuating part is considered to be acoustic) and should be eliminated by the application of filtering. In the next step the spatial distribution of the source field is investigated. Let us define the nominal size (A) of the acoustic source as the area, in which the acoustic source strength is larger than $1 \%$ of the maximum acoustic source strength. This investigation was performed for various ratios of $5 \%, 10 \%, 20 \%$ and for all cases the tendencies were the same. The nominal size of the acoustic load was the smallest in the case of the Lighthill tensor. In the case of Lamb vector formulation the source was larger both in size (A) ( $\sim 30$ times) and magnitude $\left(S_{\max }\right.$, the maximal load) ( $\sim 2$ times). This does not mean unambiguously that the usage of Lamb vector produces higher acoustic pressure in the far field, because the phase relationships also influence the final results. This observation could mean that the resolution of the source could be better in the case of Lamb vector formulation and it could be used more effectively on a coarser grid as well. In the case of time derivative of the pressure formulation the size of the acoustic source was much larger (3-4 orders of magnitude), while its magnitude was much smaller. Furthermore, it can be stated that the spatial distribution of the sources looked very similar in the compressible and incompressible simulations.

\section{Acoustic simulation}

4.1. The acoustic simulation domain. The significant advantage of the hybrid approach is that the acoustic simulation domain can be larger than the CFD domain. 


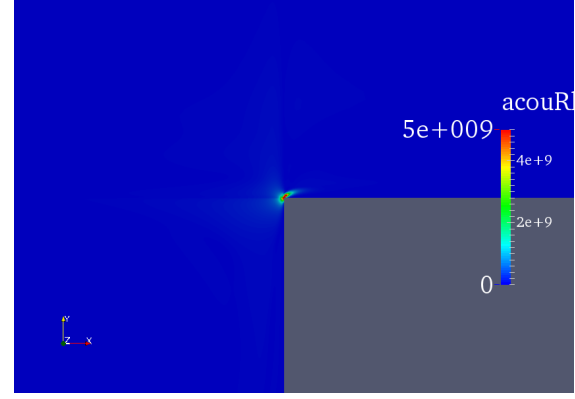

(a)

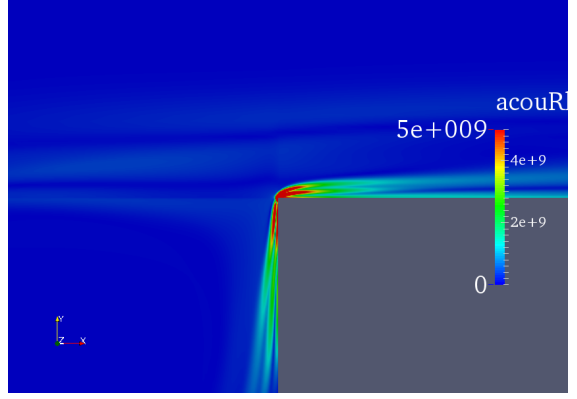

(b)

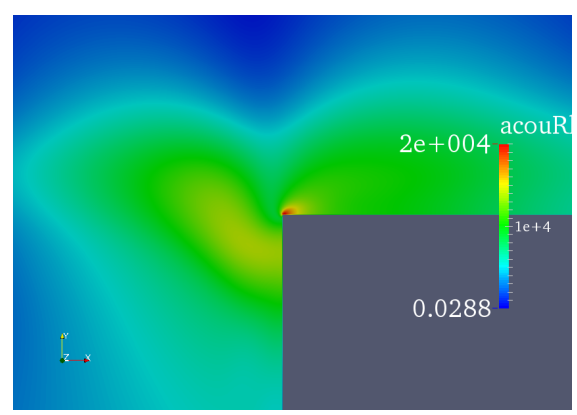

(c)

Figure 5. Spatial distribution of the acoustic source $(\mathrm{Pa} / \mathrm{m} 2)$ at the fundamental frequency. (a) divergence of the Lighthill tensor, (b) Lamb vector, (c) Second time derivative of the pressure

Table 3. Comparison of source terms defined in the incompressible flow field

\begin{tabular}{|c|c|c|c|}
\hline Name & $\frac{F_{\text {Mean }}}{F_{1 s t}}$ & $\mathrm{~A}\left[\mathrm{~m}^{2}\right]$ & $S_{\text {max }}\left[\mathrm{Pa} / \mathrm{m}^{2}\right]$ \\
\hline Lighthill & $\sim 66 \%$ & $2.519 \mathrm{e}-8$ & $5.6 \mathrm{e} 10$ \\
\hline Lamb & $\sim 133 \%$ & $6.116 \mathrm{e}-7$ & $1.5 \mathrm{e} 11$ \\
\hline Time derivative & $<1 \%$ & $8.072 \mathrm{e}-4$ & $2.1 \mathrm{e} 4$ \\
\hline
\end{tabular}

The acoustic domain contains three parts in our case. The first one is a small source region where the sound is generated. Here, the acoustic sources were calculated based on the flow simulation data and interpolated to the acoustic source grid. In this case the two meshes (the CFD and acoustic mesh) were identical. The next part is the propagation region, where the acoustic waves propagate without any amplification or damping. Within this region, the right-hand side of the acoustic wave equation is zero. This region was defined as $1 \mathrm{~m}$ before, after and above the cavity. The grid size 
here was much coarser $(5 \mathrm{~mm})$ and that is why an interpolation technique described in [16] was used at the border of two regions. The outermost part is the absorption region. This region was a thin layer at the border of simulation domain, where the waves were strongly damped to avoid reflections. Here the so-called perfectly matched layer was defined. Further information about the technique and implementation can be found in [17.

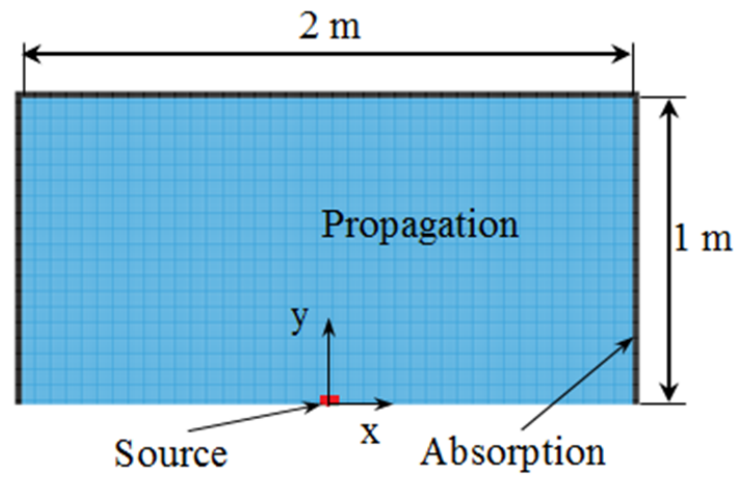

Figure 6. Parts of acoustic simulation domains: the source region, propagation and absorption region; and microphone positions

4.2. The results of the acoustic simulation domain. The acoustic simulations were carried out by CFS++, which is an in-house code at Vienna University of Technology to solve partial differential equations by the finite element method. First, the simulations based on the incompressible acoustic source formulations were carried out. A typical acoustic field of the simulation, which was calculated based on the Lighthill tensor, can be seen in Figure 7. The directivity pattern can be clearly associated to a dipole. At the same time the results provided by the second time derivative of the pressure show an absolutely different radiation pattern, as displayed in Figure 8

Howe investigated extensively the cavity tone with the same length-depth ratio at low Mach numbers [18. He calculated the acoustic field by Green functions. According to his result, the monopole characteristics of the sound appear only at higher frequencies and not at the fundamental frequency (which dominates in the time domain), if the Mach number is low. In Figure 9 the directivity patterns were plotted for different Strouhal numbers.

The monopole part of the sound source plays a significant role only if St $\gtrsim 20$ and $\mathrm{Ma}=0.01$, as well as for $\mathrm{St} \gtrsim 2$ and $\mathrm{Ma}=0.1$ according to Howe. Pure dipole pattern was observed also in 19 , where the sound field was calculated based on experimental data. The directivity pattern can indicate the validity of the source term formulations. FFT was performed in all cases on the whole acoustic pressure field (Figure 10). Good qualitative agreement can be seen with the results of Howe (Figure 9) in Figure 10 


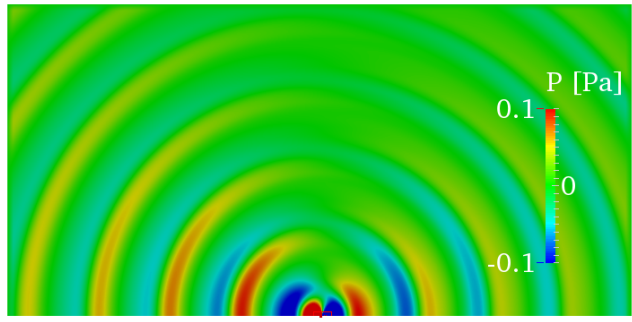

Figure 7. The instantaneous acoustic pressure field based on Lighthill analogy (incompressible)

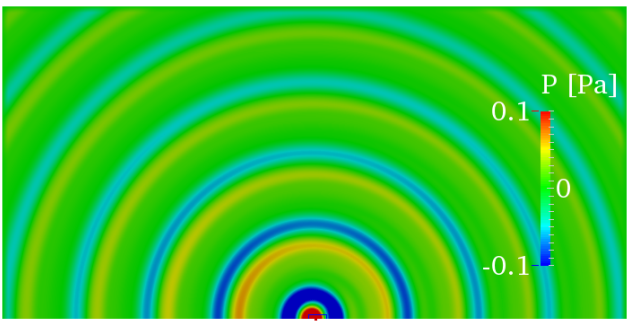

Figure 8. The instantaneous acoustic pressure field based on second time derivative of the pressure (incompressible)

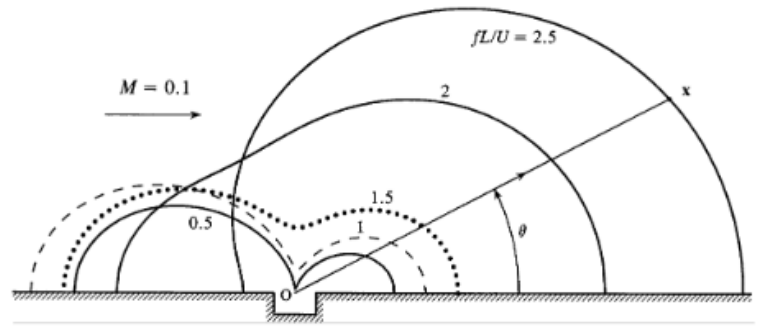

Figure 9. Directivity patterns at $\mathrm{Ma}=0.1$ and $\mathrm{St}=0.5,1,1.5,2,2.5[18$

In Figure 11, the directivity is evaluated on a circle, with a radius $r=0.4 \mathrm{~m}$ around the trailing edge. It can be seen that the velocity-based analogies ("Lighthill tensor", "Lamb vector") reproduce a dipole radiation better than the pressure-based ones. In the cases when the sources are based on the Laplacian of the pressure or the second time derivative of the pressure, the directivity patterns were unrealistic if 


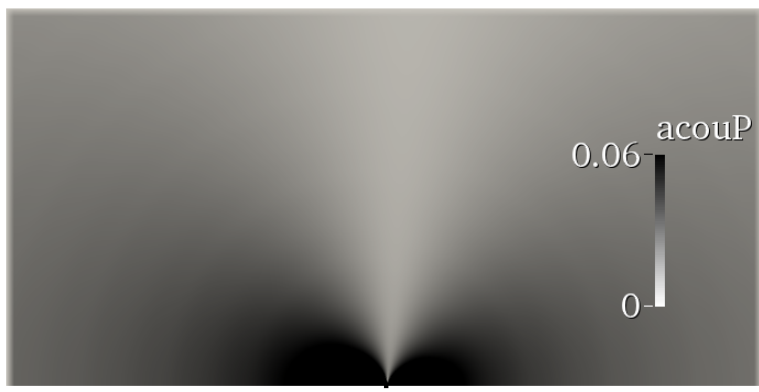

Figure 10. The FFT of the acoustic pressure field at the fundamental frequency based on Lighthill-analogy (incompressible).

we accept the correctness of the dipole pattern. The worst result was provided by the second time derivative of the pressure. In this case the sound pressure was low in upstream and downstream directions, while it was too strong above the cavity. In the case of Laplacian of the pressure, the results show the weak dipole characteristics superposed on monopole sound characteristics. Here, the amplitudes of the waves were in the correct range in both the upstream and downstream directions. The magnitudes differ slightly between its velocity-based results but this difference was negligible compared to previous cases.

The same investigation was performed on results based on sources calculated from the compressible flow simulation. The acoustic field is very similar to the incompressible cases except for the simulation based on the second time derivative of the pressure. Here a high-frequency noise appeared in the simulation. The radiation pattern looks like a dipole, while in the incompressible case it is a monopole. However, it has to be noted that the formulation is only valid for incompressible flows. It is noticeable that there are no visible differences in the source terms based on compressible and incompressible simulations (not shown here). After performing FFT on the results, the directivity patterns were also evaluated along the same circle as before.

In Figure 12, it can be seen that the results were similar to those for the incompressible cases. The main difference appeared in the pressure-based cases, which are not valid for compressible flow simulation. Here, the calculations based on the Laplacian of the pressure overpredict the sound pressure, as expected, because only the incompressible part of pressure fluctuation induces the acoustic field. At the same time the directivity pattern became more realistic in both pressure-based cases. The reason for this is unclear; maybe the different pressure computation techniques cause this huge difference, especially in the case of the time derivative of the pressure. The pressure signals at various microphone positions obtained for compressible and incompressible simulations were compared. In the case of the velocity-based formulations the differences were negligible, which was expected as these analogies are valid both in compressible and incompressible flows. 


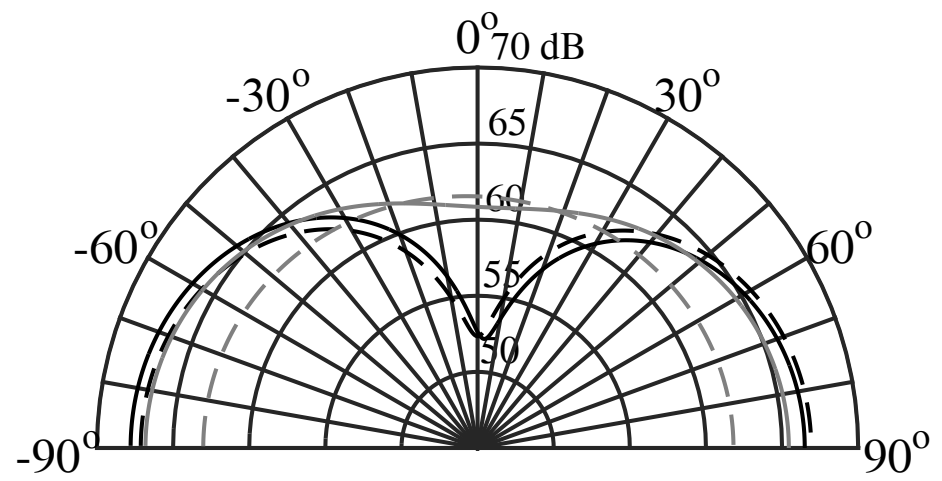

Figure 11. The directivity of the sound field at the fundamental frequency, sources are calculated based on incompressible data (black, cont.- Lighthill; black dashed - Lamb; grey, cont. - Laplacian of the pressure; grey, dashed - second time derivative of the pressure)

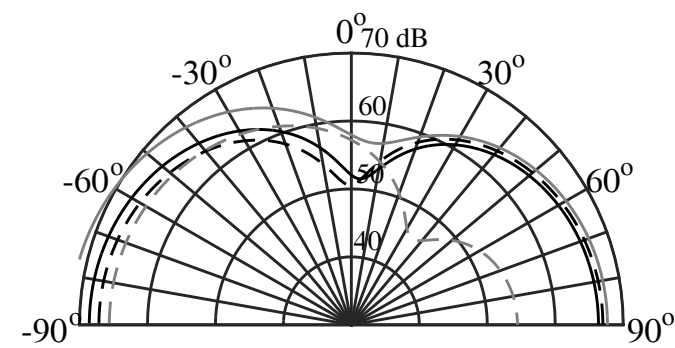

Figure 12. The directivity of the sound field at the fundamental frequency, sources are calculated based on compressible data (notation are the same as in Figure 11, )

\section{Conclusions}

In this paper various acoustic source term formulations were compared in the case of a rectangular cavity. Based on the results the following conclusions can be drawn. The velocity-based analogies provide better results than the pressure-based analogies 
with respect to the directivity patterns. The Laplacian of the pressure calculation method provides good results in upstream and downstream directions but strongly overestimates the acoustic pressure over the cavity. At the same time, no significant difference was noticed close to the trailing edge during the source term investigation in Section 2 between the second divergence of the Lighthill tensor and the Laplacian of the pressure. The second time derivative of the pressure predicts only the sound pressure in the right order of magnitude. The directivity of the sound field is wrong and the method highly overestimates the sound pressure level at the higher harmonics. It must be mentioned that the mesh study was performed only for the Lighthill analogy-based formulation. The authors are sure that such a big difference cannot be caused by a poor resolution of the acoustic sources in the case of pressure-based sources. The acoustic sources were also calculated for the compressible medium. Here, only the velocity-based analogies are valid, which was confirmed by the results, in spite of the fact that the compressibility does not play a significant role. To summarize the results, the analogy based on the Lamb vector seems to be the best. It provided the finest spatial resolution of the source along the velocity-based analogies on a same mesh, while it provides similar results to the original analogy based on Lighthill tensor. The only drawback is that the mean value was high but this can be avoided by filtering.

\section{REFERENCES}

1. Healy, G. J.: Measurements and analysis of aircraft far-field aerodynamic noise. NASA, Contractor Rep. 2377, 1974.

2. Rowley, C. W. and Williams, D. R.: Dynamics and control of high-Reynolds-number flow over open cavities. Annual Review of Fluid Mechanics, 38, (2006), 251-276.

3. YU, Y. H.: Measurements of sound radiation from cavities at subsonic speeds. Journal of Aircraft, 14, (1977), 838-843.

4. Heller, H. H. and Dobrzynski, W. M.: Sound radiation by aircraft wheel well/landing gear configurations. Journal of Aircraft, 14, (1977), 768-774.

5. Farkas, B., PaÁl, G., and Szabó, K. G.: Descriptive analysis of a mode transition of the flow over an open cavity. Physics of Fluids, 24, (2012), Paper Number 027102.

6. Gloerfelt, X.: 'Cavity noise'. Chap. 0, VKI Lectures: Aerodynamic noise from wallbounded flows. Von Karman Institute, France, 2009.

7. Hüppe, A.: Spectral Finite Elements for Acoustic Field Computation. Shaker Verlag GmbH, Aachen, 2014.

8. Kaltenbacher, M.: Numerical Simulation of Mechanic Sensors and Actuators: Finite Elements for Multiphysics. 3rd edn., Springer, Berlin, 2015.

9. Lighthill, M. J.: On sound generated aerodynamically I. General thory. Proceedings of the Royal Society of London, 211, (1951), 564-587.

10. FARKas, B. and PAÁL, G.: Numerical study on the flow over a simplified vehicle door gap - an old benchmark problem is revisited.

11. ANSYS CFX Reference Guide Release 14.5. U.S.A., 2012. 
12. FARKAS, B. and PAÁL, G.: Computational investigation on the oscillation frequencies of the shear layer ove an open cavity. Conference on Modelling Fluid Flow. The 14th International Conference on Fluid Flow Technologies, Budapest, Hungary, 2009.

13. SснӧNRоск, O.: Numerical prediction of flow induced noise in free jets of high mach numbers. Thesis of Doctor of Engineering Sciences, University of Stuttgart, 2009.

14. Richards, S., Zhang, X., Chen, X., and Nelson, P.: The evaluation of non-reflecting boundary conditions for duct acoustic computation. Journal of Sound and Vibration, $\mathbf{2 7 0}(3)$, (2004), 539-557.

15. Ashcroft, G., Takeda, K., and Zhang, X.: A numerical investigation of the noise radiated by a turbulent flow over a cavity. Journal of Sound and Vibration, 265(1), (2003), 43-60.

16. Triebenbacher, S., Kaltenbacher, M., Flemisch, B., and Wohlmuth, B.: Applications of the mortar finite element method in vibroacoustics and flow induced noise computations. Acta Acustica united with Acustica, 18, (2010), 536-553.

17. Kaltenbacher, B., Kaltenbacher, M., and Sim, I.: A modified and stable version of a perfectly matched layer technique for the $3 \mathrm{D}$ second order wave equation in time domain with an application to aeroacoustics. Journal of Computational Physics, 235, (2013), 407-422.

18. Howe, M. S.: Wall-cavity aeroacoustics at low Mach number. Tech. Rep. AM 04-001, Boston University, College of Engineering, 2004, URL http://www.dtic.mil/dtic/tr/ fulltext/u2/a419580.pdf.

19. Koschatzky, V., Westerweel, J., and Boersma, B. J.: Comparison of two acoustic analogies applied to experimental piv data for cavity sound emission estimation. 16th AIAA/CEAS Aeroacoustic Conference, 2010. 